\title{
Linfedema de miembro superior secundario al tratamiento de cáncer de mama
}

\author{
Juan Antonio Pérez P, Christian Salem Z, Enrique Henning L, \\ Fernando Uherek P y Carlos Schultz $O$.
}

\begin{abstract}
RESUMEN
El linfedema de miembro superior es una complicación común del tratamiento del cáncer de mama que afecta aproximadamente al 20 a 25\% de las pacientes. Las enfermas pueden padecer desde un aumento de volumen, dolor, tensión y pesadez en el miembro comprometido, llegando hasta la invalidez de éste. A menudo presentan infecciones cutáneas recurrentes. Por lo general este linfedema produce efectos lamentables en la calidad de vida de la mujer, sin embargo como se trata de una complicación no letal, recibe poca atención y se investiga menos que otras complicaciones del tratamiento del cáncer mamario. La etiología de linfedema es multifactorial y aunque está estrechamente relacionado con la linfadenectomía axilar y la radioterapia, aún se desconocen otros factores que contribuyen al establecimiento de esta condición y la naturaleza de la interacción de estos factores. No existe consenso respecto a la precisión de los métodos para evaluar el grado de edema del miembro superior aquejado y aún no se han desarrollado métodos exactos para evaluar el impacto funcional de esta complicación. El linfedema representa un problema de difícil manejo incluso con el advenimiento de modalidades del tratamiento modernas. Cuando se presenta inmediato a la terapia, suele resolverse durante las primeras semanas o meses del postoperatorio en un buen número de casos pero el crónico y tardío es de difícil solución. Algunas de las terapias actualmente en uso incluyen la elevación del miembro afectado, drenaje linfático manual, mangas elásticas, compresión neumática intermitente y tratamientos descongestionantes. Los tratamientos farmacológicos y quirúrgicos son controvertidos. (Palabras claves/Key words: Linfedema/ Lymphedema; Cáncer de mama/Breast cancer; Linfedema postratamiento del cancer de mama/ Lymphedema following the treatment of breast cancer).
\end{abstract}

\section{INTRODUCCIÓN}

Linfedema es la acumulación de linfa en los espacios intersticiales, principalmente en la grasa subcutánea, causado por un defecto del sistema linfático. Se trata de un desbalance entre la filtración capilar y el drenaje linfático que ocasiona un edema crónico rico en proteínas.

El linfedema que se presenta en la mujer tratada por cáncer de mama es motivado por la acumulación de linfa, un líquido rico en proteínas, en el miembro superior, producto de la disección axilar nodal, lo cual involucra una interrupción del drenaje linfático axilar, y de la terapia radiante 0 del concurso de ambas ${ }^{1,2}$. Este trastorno es quizás la secuela más desafortunada y una de las más temidas del tratamiento de cáncer de mama.

La complicación más grave del linfedema de larga evolución, aunque poco frecuente, es la degeneración hacia un linfangiosarcoma (síndrome de Steward-Treves) ${ }^{3}$.

La incidencia de linfedema varía ampliamente en los diferentes estudios ${ }^{4}$, lo cual 
se explica por la variabilidad en la evaluación de diversos factores como la extensión de la disección axilar, el intervalo entre la linfadenectomía axilar y la valoración del linfedema, los métodos usados para definir linfedema y la calidad y extensión del seguimiento de las pacientes.

Si se considera la variedad de criterios para definir el linfedema y la diversidad de técnicas de valoración, no es sorprendente observar una amplia variación en la incidencia, informándose tasas entre el $6 \%$ a $70 \%$ entre las pacientes tratadas por cáncer de mama ${ }^{5,6}$.

En general se acepta que aproximadamente $20 \%$ a $25 \%$ de pacientes tratadas por carcinoma mamario desarrollarán esta complicación, ocasionándoles algún grado de deformidad, incomodidad, e incluso invalidez del miembro superior afectado. Se desconoce por qué algunas mujeres presentarán edema y otras no. Algunas pacientes viven muchos años sin esta complicación y desarrollan repentinamente un edema masivo sin causa aparente.

\section{FISIOPATOLOGÍA DEL LINFEDEMA}

La linfa es un fluido rico en proteínas que normalmente drena desde los espacios tisulares a través de una red de linfáticos de paredes finas, hacia los linfonodos regionales, los que finalmente vacían su contenido en el sistema venoso. EI linfedema es el resultado de la acumulación de linfa en los tejidos blandos provocada por una sobrecarga funcional de los linfáticos, donde el volumen de la linfa excede la capacidad de transporte del sistema linfático.

Se puede clasificar en primario y secundario.

El linfedema primario es infrecuente y se produce por ausencia congénita de tejido linfático o por anormalidades del desarrollo del sistema linfático, pudiéndose presentar en etapas tempranas o tardías de la vida.

El linfedema secundario es más común y se origina, entre otras causas, por cirugías, irradiación e infección. En estas situaciones el transporte de la linfa se interrumpe a causa de una ruptura física, compresión u obstrucción de los conductos linfáticos ${ }^{2}$.

Las pacientes con cáncer de mama pueden desarrollar linfedema como secuela de la resección quirúrgica de linfonodos axilares, de vasos linfáticos y, además, por la fibrosis inducida por la radioterapia en la axila, circunstancias en que se obstruyen las vías de drenaje linfáticas del miembro superior. La resultante es la incapacidad de movilizar las proteínas del líquido intersticial o componente extravascular del compartimiento extracelular. La acumulación de macromoléculas en el intersticio eleva la presión oncótica tisular, mientras que la ruptura y bloqueo de los linfáticos eleva la presión hidrostática en el interior de los linfáticos restantes. Ambas situaciones contribuyen para generar edema tisular. El nivel aumentado de proteínas no sólo atrae fluido al interior de los tejidos, sino que también provoca inflamación y fibrosis dentro de ellos.

El estasis del líquido rico en proteínas combinado con un drenaje dañado de la extremidad desprovista de linfonodos, proporcionan un excelente medio de cultivo para el desarrollo de infecciones recurrentes como linfangitis y erisipela ${ }^{7}$. La linfangitis tiene una instauración rápida y se manifiesta en forma de pequeños cordones rojizos que afectan los conductos linfáticos de la extremidad, son dolorosos y presentan signos locales de inflamación asociados a fiebre. La erisipela se diferencia de la linfangitis por la aparición de placas cutáneas de color rojo, dolorosas, con calor local y frecuente presencia de adenitis. EI cuadro se inicia bruscamente en forma de malestar general, escalofríos y fiebre superior a $40^{\circ} \mathrm{C}$. El germen involucrado con mayor frecuencia es el estreptococo betahemolítico.

Las infecciones repetidas agravan la fibrosis y causan mayor daño al miembro afectado. Con la dilatación de los linfáticos las válvulas se hacen incompetentes causando mayor estasis. Hay que destacar que los compartimentos musculares bajo la fascia profunda no se afectan, probablemente por la contracción muscular dentro de un espacio de volumen fijo, la cual evita la acumulación de fluidos.

También puede producirse linfedema de miembro superior por metástasis nodales y/o invasión de tejidos blandos que causan oclusión de linfáticos.

\section{MANIFESTACIONES CLÍNICAS}

El linfedema suele empezar insidiosamente después de la disección axilar en períodos variables de tiempo. La inflamación puede variar desde una condición de escasa importancia, de severidad leve y escasamente notorio en las fases tempranas, hasta hacerse extrema en etapas posteriores, provocando un acentuado aumento de volumen, pérdida funcional variable e incluso invalidez del miembro comprometido (Figura 1). 


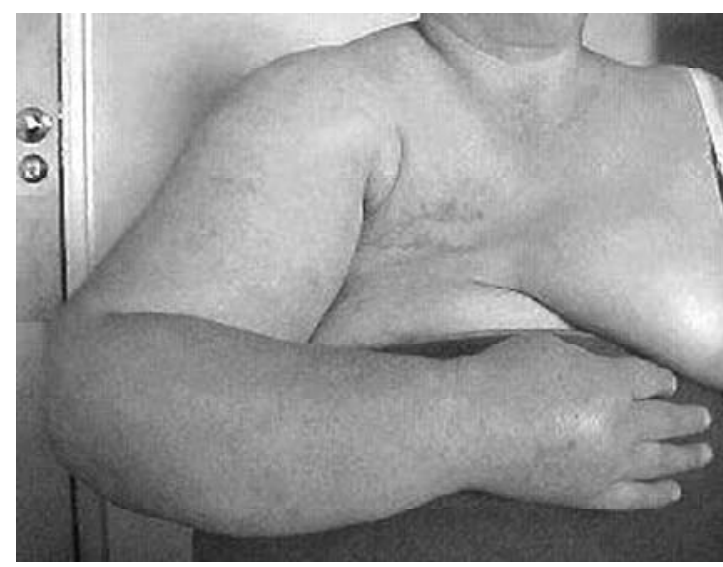

FIGURA 1. Paciente con linfedema de miembro superior secundario a mastectomía radical modificada más radioterapia postoperatoria.

Durante el período postoperatorio inmediato puede observarse cierto grado de edema del miembro superior, el cual habitualmente regresa espontáneamente en el lapso de algunas semanas o meses. Sin embargo, la inflamación puede persistir en forma crónica o puede aparecer muchos meses o años posterior al tratamiento del cáncer mamario.

La deformidad cosmética no puede ocultarse con el vestuario habitual, provoca incomodidad física y se asocia con episodios recurrentes de infecciones.

Se han descrito tres etapas de linfedema ${ }^{8,9}$.

Etapa I. Se presenta como edema blando que deja fóvea a la presión y se considera reversible.

Etapa II. Al progresar el edema éste se torna indurado, fibrótico, nofoveolar y es refractario al tratamiento.

Etapa III. Es un linfedema avanzado que presenta induración cartilaginosa de la piel, debido a la fibroesclerosis del tejido superficial y de la hipodermis, acartonamiento y formaciones verrugosas. Con los episodios repetidos de celulitis y linfangitis, la piel se torna más indurada, acartonada e hiperqueratósica.

Además de estos síntomas físicos, las pacientes pueden experimentar gran angustia y falta de comprensión motivada involuntariamente por médicos, cuyo enfoque primario es evitar y/o detectar la recurrencia del cáncer y que suelen minimizar la importancia del linfedema debido a su carácter no fatal. No obstante, para la paciente, la apariencia del brazo edematizado puede ser más agraviante que la misma mastectomía, puesto que la ausencia de la mama puede disimularse con relativa facilidad mientras que un miembro superior desfigurado es una evocación constante del cáncer de mama y constituye un motivo de curiosidad para las personas con las cuales la paciente se relaciona ${ }^{10,11}$.

\section{FACTORES ETIOLÓGICOS CIRUGÍA}

La linfadenectomía axilar es la principal causa de linfedema del miembro superior. Los argumentos para realizar la linfadenectomía incluyen estadificar el cáncer de mama, conocer el pronóstico, planificar la terapia adyuvante y minimizar la recurrencia axilar. La incidencia de linfedema es menor cuando el tratamiento quirúrgico axilar es menos agresivo, en una curva decreciente desde la mastectomía radical hasta el tratamiento conservador.

La disección axilar ha sido parte de la terapia estándar del carcinoma de mama desde fines del siglo XIX. En la mastectomía radical clásica virtualmente todos los linfonodos son resecados, junto a la mama y los músculos adyacentes a la pared torácica. Con el transcurso del tiempo las operaciones se han ido progresivamente tornando menos extensas ${ }^{12}$. En la actualidad, la disección axilar habitual incluye los niveles I y II y solamente se extiende la disección hasta incluir el nivel III si se encuentran linfoadenopatías metastásicas ${ }^{1}$ (Figura 2). Los esfuerzos están encaminados a lograr una armonía entre la extensión de la disección axilar y la magnitud de la enfermedad que se está tratando.

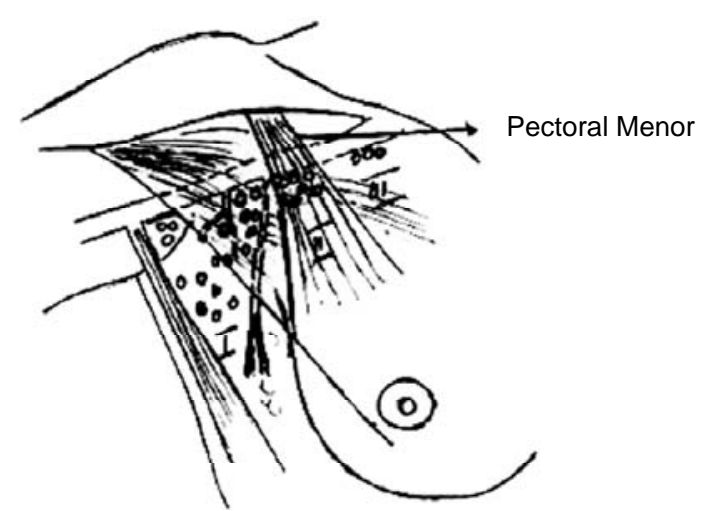

FIGURA 2. Niveles de linfonodos axilares identificados en relación a músculo pectoral menor. 
Considerando los riesgos de linfedema, en nuestros días, el cirujano diseca meticulosamente el contenido de la axila por debajo de la vena axilar, realizando disecciones y hemostasia cuidadosas y conservando el tejido graso axilar medio, lateral y superior a la vena, debido a que en este tejido existen troncos linfáticos importantes (Figura 3).

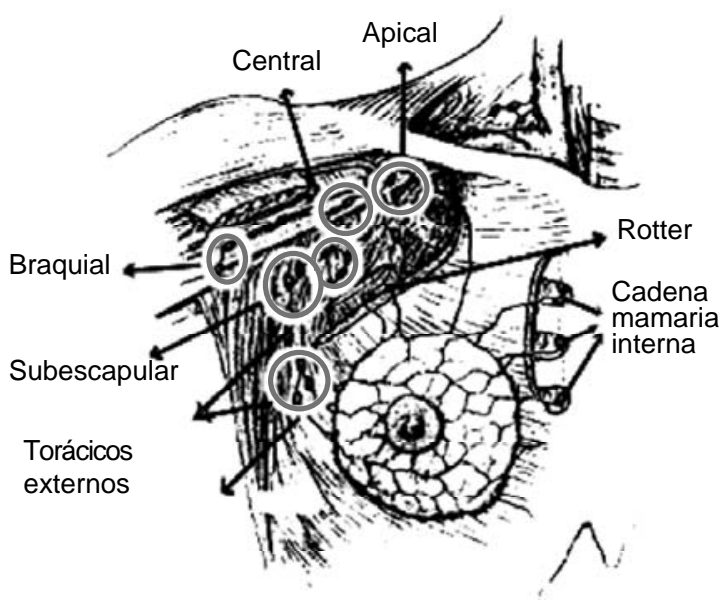

FIGURA 3. Linfonodos axilares y cadena mamaria interna

\section{RADIOTERAPIA}

Varios estudios que han evaluado la relación entre linfedema y la magnitud de disección quirúrgica, han observado que la radioterapia adicional en la axila disecada aumenta considerablemente la incidencia de linfedema ${ }^{13,14}$.

Es conocido que cuando se intenta irradiar sólo la mama (como ocurre en mastectomía parcial exclusiva), alguna dosificación de la radiación alcanza al nivel I e incluso al nivel II de la axila, dependiendo de la técnica de la radioterapia usada y de la anatomía de la paciente.

Para realizar una técnica de radiación precisa, se recomienda marcar el límite quirúrgico con clips radiopacos, con el objeto de precisar los límites de la disección axilar. El radioterapeuta puede ver el área disecada en las películas de simulación e irradiar con mayor exactitud.

\section{BIOPSIA DEL LINFONODO CENTINELA}

La incorporación de la técnica biopsia del linfonodo centinela debería disminuir el riesgo de linfedema. Si se extirpan cuidadosamente sólo uno o dos linfonodos, se puede presumir que no ocurrirá linfedema. Sin embargo, la biopsia no siempre es tan limitada o el o los linfonodos centinela pueden estar localizados muy altos, al nivel de la vena axilar y de los troncos linfáticos principales. La disección en ese sitio podría, teóricamente, producir linfedema. El riesgo de linfedema aún no se ha evaluado durante el seguimiento prolongado de pacientes manejadas con la técnica del linfonodo centinela.

Si a la biopsia del linfonodo centinela se agrega radioterapia axilar aumenta el riesgo de linfedema. Es importante recordar que diferentes series informan que la radioterapia de la axila sin que se realice ninguna cirugía axilar, va seguida de una incidencia del linfedema de $2 \%$ a $5 \%{ }^{15,16}$. Por consiguiente, la incidencia del linfedema será al menos ese porcentaje si se combinan biopsia del linfonodo centinela y radioterapia axilar. Se ha demostrado que el linfedema posterior a la radiación axilar exclusiva se desarrolla en forma más tardía que el de la cirugía axilar combinada con radioterapia. ${ }^{17}$

Además de estos dos factores bien establecidos, la magnitud de la disección quirúrgica y la radiación a la axila, existe una amplia gama de posibles factores etiológicos que no se han evaluado sistemáticamente ${ }^{1}$. La infección postoperatoria a menudo ha sido mencionada como un factor relevante en la génesis del linfedema ${ }^{18}$.

Algunas investigaciones han revelado datos poco convincentes y algunos contradictorios sobre otros factores, tales como la edad avanzada al momento del diagnóstico del cáncer de mama, la obesidad, la mastectomía en el lado del miembro superior dominante, la sección del músculo pectoral menor durante la mastectomía, volumen del débito del dren y formación de seroma.

\section{VALORACIÓN DE LINFEDEMA}

No hay ninguna definición operacional consistente de "linfedema clínicamente significativo". La falta de una definición produce dificultades para establecer la verdadera incidencia de esta secuela. Si bien las medidas físicas y técnicas de imágenes proporcionan una valoración cuantitativa del crecimiento del brazo, no existe ninguna medida exacta para evaluar el impacto funcional de linfedema.

No está establecido un grado de aumento de volumen estándar que constituya linfedema. Aunque el concepto más corriente es una 
diferencia de $2 \mathrm{~cm}$ entre las circunferencias de los brazos, tal hinchazón podría ser severa en un brazo delgado y no detectable en otros más gruesos. En casos raros puede considerarse una variación natural de $2 \mathrm{~cm}$ o más en la extremidad más utilizada o extremidad dominante.

Aproximadamente $50 \%$ de las pacientes con linfedema mínimo documentado $(2 \mathrm{~cm})$ sufre síntomas de "pesadez del brazo"3. Una encuesta por correo encontró que la mitad de las pacientes que presentan tal grado de linfedema no informan de este problema a ningún médico ${ }^{19}$. Por lo tanto, para diagnosticar oportunamente un linfedema, se debe inquirir información sobre síntomas de pesadez, tensión y aumento de volumen del miembro superior afectado.

Los criterios más habituales para diagnosticar y medir el linfedema son ${ }^{20,21:}$

- Mediciones circunferenciales en varios lugares de los miembros superiores, teniendo como referencias algunos puntos óseos.

- Medidas volumétricas usando sumersión del miembro en fluidos, $y$

- Tonometría de piel y de tejido blandos en la que se cuantifica la compresión del tejido blando.

El método tradicional para evaluar el linfedema es la medición mediante una huincha, tanto en el preoperatorio como en el postoperatorio, de las circunferencias en ambos miembros superiores en 4 puntos: articulaciones metacarpofalángicas, muñeca, $10-12 \mathrm{~cm}$ hacia distal de los epicóndilos laterales o del olécranon y $14-16 \mathrm{~cm}$ hacia proximal de esas referencias óseas $^{22}$. Estas mediciones se usan con mayor frecuencia, porque las cintas para medir están habitualmente disponibles y porque la medida volumétrica resulta logísticamente más difícil de realizar. Si bien las mediciones con cintas parecieran ser adecuadas, pueden variar dependiendo el grado de compresión que se ejerce con la cinta sobre el tejido blando.

Una diferencia mayor a $2 \mathrm{~cm}$ en cualquiera de estas cirfunsferencias es definida por algunos autores como "linfedema clínicamente significativo", mientras que otros clasifican este grado como linfedema leve. Cualquiera sea la denominación, este linfedema debe ser tratado, siempre que previamente se haya excluido que el tumor envuelva la axila o plexo braquial, que exista infección y/o trombosis de la vena axilar.
La medida del volumen del brazo por desplazamiento de agua es más exacta y sus resultados revelan un sólo valor, pero la técnica es embarazosa y, por ende, poco empleada.

La tonometría de piel y tejido blandos, realizado con un dispositivo de medición de presión que aprieta en la piel, no es un procedimiento estandarizado.

Otros métodos por evaluar linfedema que están en proceso de investigación son la linfocintigrafía isotópica, la resonancia nuclear magnética, la tomografía axial computada y el ultrasonido ${ }^{1}$. La linfocintigrafía isotópica se ha usado de manera preliminar para predecir qué pacientes tienen riesgo elevado de presentar linfedema después del tratamiento axilar, abrigando la esperanza de enfatizar las estrategias de prevención y la necesidad de un seguimiento frecuente.

\section{PREVENCIÓN DE LINFEDEMA}

Como no se ha encontrado un tratamiento efectivo para esta secuela, debe ponerse énfasis en su prevención. No obstante, sin un conocimiento basado en evidencia de los factores etiológicos, la lista de precauciones del miembro superior postratamiento está basado en un razonamiento intuitivo.

Es importante considerar que toda mujer tiene una anatomía de sus vías linfáticas congénitamente distinta. La linfografía isotópica permite una buena valoración funcional del flujo linfático, sin embargo la definición morfológica de las estructuras linfáticas es pobre ${ }^{7}$. Las peculiares características del sistema linfático en conjunto con el tipo de tratamiento axilar, son las determinantes principales de las estrategias preventivas.

Las medidas preventivas no deben ser rígidas y deben adaptarse a cada paciente puesto que en el caso de algunas mujeres, éstas pueden ser contraproducentes, al actuar como "sobreprotección" llevando al subuso del miembro y a la atrofia muscular.

\section{PRECAUCIONES DE LA EXTREMIDAD SUPERIOR ${ }^{1,2,23}$}

Para prevenir el edema y/o infección del miembro superior, es indispensable instruir a la paciente sobre algunas medidas referentes a cuidados que podrían evitar la aparición de linfedema.

1. Evitar inyecciones endovenosas, intramusculares o subcutáneas, administrar vacunas o 
practicar test de alergias en el lado de la mastectomía.

2. Evitar extracciones de sangre de dicho brazo.

3. Evitar tomar la presión arterial en dicho miembro.

4. Evitar dañar la piel. Debe realizarse un cuidadoso aseo de la piel y de las uñas y no cortar las cutículas. Prestar atención inmediata y curar apropiadamente cualquier lesión cutánea por mínima que sea limpiándola con agua y jabón para luego cubrirla.

5. En caso de infecciones utilizar antibióticos precozmente.

6. Evitar afeitar la axila con hojas o inyectores. Usar máquina eléctrica con el objeto de reducir el riesgo de erosiones.

7. Evitar el calor, quemaduras domésticas (cocina), quemaduras solares 0 bronceados, baños calientes y saunas. En caso de quemaduras consultar al médico.

8. Evitar el uso de mangas apretadas o puños elásticos en blusas y camisas de dormir.

9. Evitar relojes, pulseras o anillos ajustados en el brazo del lado de la operación.

10. Evitar cargar paquetes o bultos pesados.

11. Evitar la utilización de químicos irritantes y compuestos abrasivos.

12. Evitar ejercicios violentos y extenuantes. El ejercicio es importante en la rehabilitación, sin embargo, si el miembro afectado empieza a doler o se siente cansado, se debe suspender. Los ejercicios aeróbicos enérgicos del brazo se pueden efectuar sólo si se utiliza una manga de compresión.

13. Usar dedales para coser.

14. Protegerse y/o usar repelentes para evitar picaduras o mordeduras de insectos (zancudos, pulgas, abejas, etc.)

15. Usar guantes de protección para manipular cuchillos y tijeras, al cultivar un huerto o jardín y al usar detergentes fuertes 0 jabones de uso ordinario.

Todas las pacientes deben ser educadas en la misma forma en relación con los cuidados del miembro superior; sin embargo, estas precauciones pueden resultar exageradas para las enfermas con bajo riesgo de linfedema, mientras que pueden ser insuficientes para aquellas de mayor riesgo. Además, como en el linfedema pueden ocurrir varias décadas ${ }^{24}$ después del tratamiento axilar, las pacientes deben mantener este comportamiento por el resto de sus vidas.

\section{TRATAMIENTOS DE LINFEDEMA}

La práctica de no tratar el linfedema es una conducta poco acertada, aunque es bastante común. A menudo, se enfatiza a una mujer que porta esta secuela que "debe estar agradecida de estar viva" y que debe "aprender a vivir con su linfedema". Esta situación debe corregirse y hay que educar a los médicos, tanto para detectar como para reconocer los signos tempranos del linfedema, puesto que mientras más precoz se diagnostique y trate esta complicación, se requerirán menos medidas terapéuticas para impedir su progresión.

Aunque es inobjetable que el linfedema en la mayor parte de los casos es irrecuperable, una terapia precoz y perseverante puede lograr que la paciente vuelva a desarrollar la mayor parte de sus actividades de su vida normal y evitar las complicaciones que agravan su pronóstico y que pueden invalidar.

El enfrentamiento de linfedema ya establecido varía desde no hacer ningún tratamiento hasta realizar procedimientos quirúrgicos agresivos, la mayoría de ellos abandonados. Entre estas dos conductas extremas existen dos categorías de tratamiento conservador del linfedema: el mecánico y el farmacológico.

\section{TRATAMIENTO MECÁNICO}

Elevación del miembro afectado. Aunque la elevación puede ser útil, reduciendo el linfedema a través del principio de la gravedad, muchas veces resulta ser poco práctica e incómoda.

Compresión elástica. La compresión elástica entre 20 a $60 \mathrm{~mm} \mathrm{Hg}$ de presión es una efectiva medida para manejar el linfedema y puede utilizarse como terapia primaria $^{25}$. La paciente debe ajustarse una manga elástica desde la muñeca hasta la axila (Figura 4). Si es necesario se puede incorporar un guante independiente de la manga que sea removible para permitir lavarse las manos sin quitar la manga entera. Algunos clínicos recomiendan este soporte elástico en forma permanente y otros durante el día o cuando se realizan ejercicios ${ }^{26,27}$. Adicionalmente la manga protege de injurias como quemaduras, laceraciones y picaduras de insectos. Debe 
reemplazarse entre los 4 a 6 meses o cuando empiece a perder elasticidad.

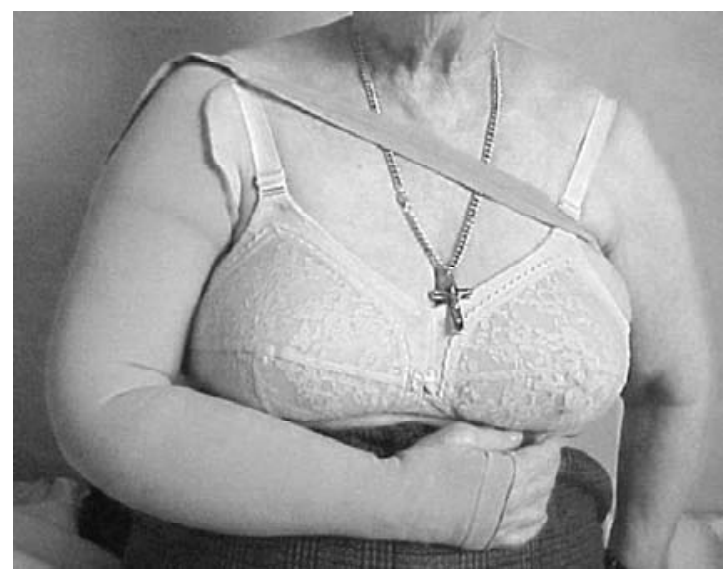

FIGURA 4. Manga elástica en miembro superior con linfedema.

La paciente debe estar advertida que estas mangas tienen algunos inconvenientes, entre ellos, son poco elegantes, producen las molestias propias de una compresión, son relativamente difíciles de ponérselas $y$, por último, son caras.

El profesional que indica este tipo de accesorios debe instruir a las pacientes sobre su apropiada aplicación. Al respecto, es frecuente que esta tarea quede confinado a un empleado de una tienda de suministros médicos que carece de entrenamiento específico.

Ejercicios. Los ejercicios activos mesurados de los músculos del brazo y el antebrazo pueden ser utilizados para activar la "bomba muscular", con la esperanza de que se logre aumentar el flujo venoso y linfático. El ejercicio se realiza mejor con un mínimo de movimientos de la extremidad, de manera que la paciente pueda hacerlos moderadamente cuando ella lo desee. De 5 a 10 repeticiones realizadas cada 5 minutos durante una hora 2 veces al día será suficiente. El ejercicio puede ser más efectivo si se hace con un soporte elástico externo. La repetición excesiva de los ejercicios puede ocasionar un exagerado incremento del flujo sanguíneo arterial en el miembro, que puede aumentar la formación del edema ${ }^{2,3}$.
Drenaje linfático manuaf8. Es una técnica de masaje que estimula los vasos linfáticos para conseguir que se contraigan con mayor frecuencia, siguiendo anatómicamente los trayectos linfáticos del cuerpo. Los masajes de drenaje linfático se inician centralmente en el cuello y en el tronco con el objeto de vaciar las vías linfáticas principales, y de esa forma facilitar el drenaje de la linfa acumulada en el miembro superior afectado.

Vendajes. Inmediatamente después del drenaje linfático manual pueden utilizarse vendajes en capas múltiples, con poca presión. Las vendas se instalan desde las yemas de los dedos hasta la axila con mayor presión distal y menos presión proximal. Se usan varias capas de vendas de algodón poco elasticadas bajo las cuales se insertan capas de moltopren para asegurar una distribución de presión uniforme o aumentar presión en áreas que son particularmente fibróticas. El paciente vendado se guía luego a través de ejercicios que involucran un rango activo de movimiento con los músculos y articulaciones que funcionan dentro del espacio cerrado del vendaje. Generalmente se evita el ejercicio isométrico.

Bombas de compresión neumática ${ }^{23}$. Se ha planteado como alternativa al masaje linfático. Existe una variedad de modelos de dispositivos, pero básicamente se tratan de dos tipos, unos que utilizan cámara única y otros más modernos que poseen cámaras múltiples. El sistema de cámara simple proporciona compresión intermitente no segmentada a lo largo de todo el brazo tratado; sin embargo, también puede ocasionar flujo retrógrado de la linfa que puede causar un aumento del linfedema en el extremo distal del brazo. Los que tienen cámaras múltiples pueden proporcionar compresión secuencial de distal a proximal, teóricamente impulsando el líquido hacia delante de su compresión. Es difícil comparar la eficacia de ambos métodos ya que no existe consenso respecto a la presión óptima de la bomba, su duración y frecuencia de las sesiones.

Fisioterapia decongestiva completa ${ }^{29}$. Este procedimiento terapéutico considera que el linfedema compromete un cuadrante completo del cuerpo, aunque sus efectos son más relevantes en el brazo y mano. 
Incluye cuidados de la piel, drenaje linfático manual, vendajes compresivos de capas múltiples con baja presión (seguido por un vestido de compresión ajustado cuando el edema se ha reducido), y ejercicios terapéuticos con los vestidos o vendas. Debe ser realizada por terapeutas especializados y especialmente entrenados.

\section{TRATAMIENTO MEDICAMENTOSO}

Antibióticos. Desde el punto de vista farmacológico, lo más importante es un tratamiento precoz de una erisipela o linfangitis mediante penicilina G sódica. También es útil la administración de penicilina retardada (penicilina benzatina, 1.200.000 UI por mes) durante largos períodos, con el fin de prevenir los brotes infecciosos, los cuales tienen una relevancia importante en el empeoramiento progresivo del linfedema ${ }^{7}$.

Diuréticos. No son eficaces para reducir edemas ricos en proteínas como es el caso del linfedema. Aunque pueden movilizar agua temporalmente, el aumento de la presión oncótica ejercida por la alta concentración de proteínas en los espacios intersticiales, produce una rápida reproducción del linfedema e incluso podrían acelerar el proceso de fibrosis.

Benzopironas. Algunas benzopironas y derivados de plantas ${ }^{7}$ (Ruscus aculeatus, Hesperidina) mejorarían el linfedema crónico a través de la activación de la proteolisis por macrófagos e induciendo un aumento del numero total de macrófagos, lo que produciría remoción del exceso de proteína en los espacios tisulares llevando a una disminución de la presión oncótica y del fluido del edema ${ }^{27}$.

Lubricantes de la piel. De especial relevancia es mantener bien lubricada la piel del miembro afectado mediante emulsiones protectoras.

\section{TRATAMIENTO QUIRÚRGICO}

La cirugía se ha planteado sólo en los casos en que han fallado todas las medidas conservadoras. Sus resultados no han sido satisfactorios. Se han propuesto numerosos procedimientos pero sus resultados han sido pobres.

En general existen 2 tipos de métodos quirúrgicos: fisiológicos y reductivos.

Los procedimientos fisiológicos pretenden restaurar el flujo linfático del miembro anastomosando los conductos linfáticos dilatados con venas utilizando técnicas de microcirugía.

Las técnicas reductoras simplemente resecan el exceso de tejido edematoso para llevar al miembro a un tamaño más funcional. Estos procedimientos realizan la remoción de piel y el tejido hipodérmico seguido por injerto de piel. Más recientemente, en Suecia, se han reportado buenos resultados a corto plazo con liposucción ${ }^{30}$ aunque la eficacia a largo plazo del procedimiento aún no es conocida.

\section{CONCLUSIONES}

El aumento de la expectativa de supervivencia de la mujer con cáncer de mama plantea interrogantes importantes sobre cómo mejorar la calidad de vida de las pacientes que presentan complicaciones secundarias a su terapia. El linfedema del miembro superior es un problema trascendental para la mujer que puede producir un importante deterioro funcional y psicológico. Esta secuela es especialmente desalentadora para mujeres a quienes las han esperanzado que su pronóstico es excelente y que podrán continuar sus vidas con escasas secuelas visibles de su enfermedad.

Las medidas preventivas son conocidas, pero no han sido bien estudiadas. La terapia con dispositivos de compresión elástica y el drenaje linfático manual han demostrado ser las terapias más eficaces para manejar el linfedema. El efecto de las terapias farmacológicas aún permanece incierto. La comparación de resultados de los estudios es difícil por el hecho que las definiciones de procedimientos y medidas de resultados y de estratificación de riesgo varían substancialmente entre los diferentes estudios. Como la prevalencia del edema del brazo es mayor a medida que aumenta la sobrevivencia de las mujeres con cáncer de mama, es indispensable implementar y evaluar la eficacia de las estrategias de prevención y de los procedimientos terapéuticos. 


\section{REFERENCIAS}

1. Petrek JA, Pressman PI, Smith RA: Lymphedema: Current issues research and management. $C A$ Cancer J Clin 2000; 50: 292-307

2. Pain SJ, Purushotham AD: Lymphoedema following surgery for breast cancer. Br J Surg 2000; 87: 1128-41

3. Stewat FW, Treves N: Lymphangiosarcoma in postmastectomy lymphoedema. A report of six cases in elephantiasis chirurgica. Cancer 1948; 1: $64-81$

4. Petrek JA, Heelan MC: Incidence of breast carcinoma-related lymphedema. Cancer 1998; 83 (Suppl12): 2776-81

5. Segerstrom K, Bjerle P, Graffman S, Nystrom A: Factors that influence the incidence of brachial edema after treatment of breast cancer. Scand J Plast Reconstr Hand Surg 1992; 26: 223-7

6. Larson D, Weinstein M, Goldberg I, et al: Edema of the arm as a function of the extent of axillary surgery in patients with stage I - II carcinoma of the breast treated with radiotherapy. Int $J$ Radiat Oncol Biol Phys 1986; 12: 1575-82

7. Jiménez Cossío JA. Trastornos de los vasos linfáticos. En: Farreras Valentí P, Rozman C (eds), Medicina Interna. Madrid, Mosby/Doyma, 1995: 662-4

8. Brennan MJ, DePompolo RW, Garden FH. Focused review:postmastectomy lymphedema. Arch Phys Med Rehabil 1996; 77: 574-80

9. Foldi E, Foldi M, Clodius L: The lymphedema chaos: A lancet. Ann Plast Surg 1989; 22: 505-15.

10. Passik SD, McDonald MV: Psychosocial aspects of upper extremity lymphedema in women treated for breast carcinoma. Cancer 1998; 83 (Suppl 12): 2817-20

11. Dorval M, Maunsell E, Deschênes L, Brisson J, Mâsse B: Long-term quality of life after breast cancer: comparison of 8-year survivors with population controls. J Clin Oncol 1998; 16: 487-94

12. Pressman PI: Surgical treatment and lymphedema. Cancer 1998; 83 (Suppl 12): 2782-7

13. Yeoh EK, Denham JW, Davies SA, Spittle MF: Primary breast cancer. Complications of axillary management. Acta Radiol Oncol 1986; 25: 105-8

14. Dewar JA, Sarrazin D, Benhamou E, et al: Management of the axilla in conservatively treated breast cancer: 592 patients treated at Institut Gustave-Roussy. Int J Radiat Oncol Biol Phys 1987; 13: 475-81

15. Aitken RJ, Gaze MN, Rodger A, et al: Arm morbidity within a trial of mastectomy and either nodal sample with selective radiotherapy or axillary clearance. Br J Surg 1989; 76: 568-71
16. Meek AG: Breast radiotherapy and lymphedema: Cancer 1998; 83 (Suppl 12): 2788-97

17. Ivens D, Hoe AL, Podd TJ, et al: Assessment of morbidity from complete axillary dissection. $\mathrm{Br} J$ Cancer 1992; 66: 136-8

18. Mozes M, Papa MZ, Karasik A, et al: The role of infection in post-mastectomy lymphedema. Ann Surg 1982; 14: 4-15

19. McCaffrey JF: Lymphedema. Its treatment. In: Paterson AHG, Lees AW (eds), Fundamental problems in breast cancer. Boston, Martinus Nijhoff Publishing, 1987: 259-63

20. Passik SD, Newman M, Brennan M, Tunkel R: Predictors of psychological distress, sexual dysfunction and physical functioning among women with upper extremity lymphedema related to breast cancer. Psycho-Oncology 1995; 4: 255-63

21. Casley-Smith JR: Measuring and representing peripheral oedema and its alterations. Lymphology 1994; 27: 56-70

22. Bertelli G, Venturini M, Forno G, Macchiavello F, Dini D: Conservative treatment of postmastectomy lymphedema: A controlled,randomized trial. Ann Oncol 1991; 2: 575-8

23. Harris SR, Hugi MR, Olivotto IA, Levine M: Clinical practice guidelines for care and treatment of breast cancer: 11. Lymphedema. CMAJ 2001; 23: 191-7.

24. Brennan MJ, Weitz J: Lymphedema 30 years after radical mastectomy. Am J Phys Med Rehabil 1992; 71: 12-4.

25. Petrek JA, Lerner R: Lymphedema. In: Harris JR, Lippman ME, Morrow M, Hellman S, (eds). Diseases of the breast. Philadelphia, LippincottRaven, 1996: 896-903

26. Harris SR, Niesen-Vertommen SL: Challenging the myth of exercise-induced lymphedema following breast cancer:a series of case reports. J Surg Oncol 2000; 74: 95-9

27. Brennan MJ, Miller LT: Overview of treatment options and review of the current role and use of compression garments, intermittent pumps, and exercise in the management of lymphedema. Cancer 1998; 83: 2821-7

28. Kasseroller RG: The Vodder School. The Vodder method. Cancer 1998; 83 (Suppl 12): 2840-2

29. Foldi M: Treatment of lymphedema. Lymphology 1994; 27: 1-5

30. Brorson H, Svensson H, Norrgren K, Thorsson $\mathrm{O}$ : Liposuction reduces arm lymphedema without significantly altering the already impaired lymph transport. Lymphology 1998; 31: 156-72 